\title{
Prevalence of Sarcopenia in Community-Dwelling Older Adults According to Simplified Algorithms for Sarcopenia Consensus Based on Asian Working Group for Sarcopenia
}

This article was published in the following Dove Press journal:

Clinical Interventions in Aging

\author{
Hee-Kyung Chang (D) \\ Ji-Yeon Lee ${ }^{2,3}$ \\ Cho-Rong $\mathrm{Gil}^{3}$ \\ Mi-Kyoung Kim (D) ${ }^{3,4}$ \\ 'College of Nursing, Senior Health \\ Research Center of the Health \& Science \\ Institute, Gyeongsang National \\ University, Jinju, Gyeongnam-do, South \\ Korea; ${ }^{2}$ Nursing Department, Samsung \\ Changwon Hospital, Changwon, \\ Gyeongnam-do, South Korea; ${ }^{3}$ Graduate \\ College of Nursing, Gyeongsang National \\ University, Jinju, Gyeongnam-do, South \\ Korea; ${ }^{4}$ Nursing Department, Hamyang \\ Center Clinic, Hamyang, Gyeongnam-do, \\ South Korea
}

Purpose: This study sought to identify the prevalence of sarcopenia in community-dwelling Korean older adults and validate two simplified diagnostic algorithms based on the Asian Working Group for Sarcopenia (AWGS) algorithm for identifying sarcopenia.

Patients and Methods: Patients $(n=338)$ aged $\geq 65$ years participated in this crosssectional study. Muscle strength was measured by hand grip strength, physical performance by gait speed, and muscle mass by the skeletal muscle mass index (SMI). Sarcopenia was assessed using the AWGS-recommended algorithm and two simplified algorithms (A and B). Algorithms A and B were validated with respect to the AWGS-recommended algorithm using the chi-square test, and the sensitivity and specificity were obtained.

Results: Sarcopenia prevalence, determined using the AWGS-recommended algorithm, was $40.3 \%$ and $41.3 \%$ in men and women, respectively. The overall prevalence of sarcopenia was $41.0 \%$ by the AWGS-recommended algorithm, $37.6 \%$ by algorithm $\mathrm{A}$, and $37.6 \%$ by algorithm B; 111 participants were diagnosed with sarcopenia using all three methods $(p=0.157)$.

Conclusion: We established sarcopenia prevalence among rural community-dwelling adults in Korea and confirmed that the simplified algorithms were suitable for the identification of sarcopenia in rural community-dwelling older adults in Korea. Further studies are needed to assess whether these simplified algorithms are applicable to older Asian adults with functional and/or cognitive impairment and nursing home residents.

Keywords: algorithm, Asian Working Group for Sarcopenia, AWGS, elderly, sarcopenia, validation

\section{Introduction}

According to Korea's 2019 statistics, the population of elderly individuals, aged 65 years and older, accounts for $14.9 \%$ of the total Korean population. This proportion is expected to increase to $43.9 \%$ by 2060 , reaching the highest level of a super-aged society. ${ }^{1}$ With the aging of the society, interest in geriatric diseases has been increasing. In particular, interest in the prevention of and intervention for sarcopenia, which is known as an important risk factor for geriatric syndromes, has escalated. $^{2}$ Sarcopenia involves the deterioration of skeletal muscle tissue with age and is one of the most important causes of functional decline and the loss of independence in older adults, ${ }^{3}$ increasing the risk of functional degradation, fall,
Graduate College of Nursing, Gyeongsang National University, 816-15, Jinju-Daero, Jinju, Gyeongnam 5227, South Korea

Tel +82-10-8554-8985

Fax+82-55-772-8222

Email yeonmk@gnu.ac.kr 
hospitalization, and mortality. ${ }^{4}$ Consequently, there is a need for the active prevention and management of sarcopenia in community-dwelling older adults.

A systematic review has reported that the estimated prevalence of sarcopenia varies from $9.9 \%$ to $40.4 \%$, depending on the definition used. ${ }^{5}$ These differences in prevalence also exist across populations even when using the same definition. The prevalence of sarcopenia was $63 \%$ in 249 patients in long-term care facilities in Spain, based on the first published algorithms of the European Working Group on Sarcopenia in Older People (EWGSOP). ${ }^{4}$ In addition, Chinese studies showed that $19.31 \%$ of 4500 community-dwelling individuals $>50$ years old (mean age $62.4 \pm 8.3$ years) had sarcopenia, based on the Asian Working Group for Sarcopenia (AWGS) algorithm. ${ }^{6}$ As such, the prevalence of sarcopenia varied according to the criteria used and race investigated. However, a sarcopenia diagnosis remains challenging because there is no simple and rapid diagnostic standard suitable for use in community-dwelling elderly.

Baumgartner et al, ${ }^{7}$ who first defined sarcopenia, presented it as only a reduction in muscle mass. In 2010, the EWGSOP, the International Working Group on Sarcopenia (IWGS), and the AWGS presented a diagnostic algorithm for sarcopenia that included three types of gait speed (GS), hand grip strength (HGS), and muscle mass. However, the cut-off points of the diagnostic criteria of the EWGSOP and the AWGS among the sarcopenia diagnostic algorithms differ. Although diagnostic criteria based on GS used the same cut-off points, the thresholds for HGS and muscle mass differed. ${ }^{3}$ In fact, HGS in Asians is $25 \%$ lower than in people of white origin and is $27 \%$ lower in women than in men because Asians have a relatively slender body shape compared to Westerners, and sarcopenia in Asians involves a relatively small muscle mass. ${ }^{3}$ Therefore, there is a need to develop diagnostic criteria for sarcopenia that is suitable for Asians and particularly for community-dwelling older adults in Korea.

Two simplified algorithms, ${ }^{4}$ based on the AWGS criteria, ${ }^{3}$ have been reported. Because the simplified algorithm is only EWGSOP standard, it is the first time in Asia to implement simplified algorithms based on AWGS. These simplified algorithms allow rapid diagnosis, do not have a significant impact on the existing prevalence rate, and do not require the measurement of GS as GS assessment may not affect the diagnostic outcome. ${ }^{8,9}$ Originally, GS was included in the AWGS algorithm because it was considered the simplest and most reliable way to diagnose sarcopenia. However, among hospitalized and communitydwelling residents, there are many older adults who cannot walk well, and the risk of falls is very high due to space restrictions during the measurement of GS. These simplified algorithms would thus be a suitable tool for assessing sarcopenia in Asian community-dwelling older adults, but they have not yet been validated to date.

Therefore, this study sought to investigate the prevalence of sarcopenia in Korean community-dwelling older adults by using these algorithms. Concurrently, the prevalence of sarcopenia using the two simplified algorithms, without GS, and that determined using the AWGSrecommended algorithm, including GS, were compared, to evaluate the necessity of including GS when screening for sarcopenia.

\section{Patients and Methods}

\section{Study Design and Participant Recruitment} We recruited community-dwelling older adults via an advertisement at a community centre and a clinic located in Hamyang, a rural area in Korea. The inclusion criteria were adults aged over 60 years, who lived in the community and were able to walk independently. Subjects with a verified frailty status, such as wearing a pacemaker and having severe cardiac, pulmonary, or musculoskeletal disorders, and subjects who were receiving prescribed medications, such as long-term steroid therapy, which is known to affect changes in body composition, were excluded. Individuals who could not communicate with the others due to mental disorders, severe cognitive impairment and severe hearing problems were also excluded from this study. Data were collected through questionnaires and physical measurements from October 2019 to March 2020. Of the 400 participants, 12 were excluded from this study due to missing data; therefore, we collected and analyzed data for the remaining 388 .

The trained clinical research assistants visited all participants in various places in which elderly people in rural areas resided, such as the senior community center, the welfare center, and the elderly university in Hamyanggun. They then briefly explained the purpose and content of this study, and recruited subjects who met inclusion standards and collected data. The clinical research assistant recorded characteristics, such as sex and age, and clinical data, such as the use of walking aids, if any, and cognitive function, using a general questionnaire. Cognitive function was measured using the Korean 
version of the mini-mental state examination (MMSE$\mathrm{K}) ;{ }^{10}$ older adults with severe cognitive impairment were screened. The anthropometric variables of all participants were checked. Height was measured with a portable extensometer (InLab550; Biospace Co., Ltd., Seoul, Korea), which measures the length through an ultrasonic sensor. Height was measured to the nearest $0.5 \mathrm{~cm}$ and weight was measured to the nearest $0.1 \mathrm{~kg}$. Body mass index (BMI) was calculated as the ratio of the weight to the height squared. $\left(\mathrm{kg} / \mathrm{m}^{2}\right)$

This study was approved by the Ethics Committee of the Gyeongsang National University and written informed consent was obtained from each participant. The study design and employed procedures were in accordance with ethical standards and the Declaration of Helsinki. Each participant was fully informed about the risks associated with study participation and gave their written informed consent.

\section{Data Collection}

In this study, an indicators that can indirectly measure sarcopenia defined by the AWGS were chosen as variables. Muscle strength was measured by HGS, physical performance was measured by GS, and muscle mass was measured by the skeletal muscle mass index (SMI).

\section{Hand Grip Strength}

HGS was measured with a CAMRY hand grip dynamometer (CAMRY EH101; Henqi, Guangdong, China). Participants were instructed to squeeze the dynamometer as hard as they could. Both hands were tested with the participant in a seated position, with their elbow flexed at a 110 degree angle, their wrist placed in a neutral position, and their interphalangeal joint of the index finger positioned at a 90 angle. Two readings were obtained for each hand, and the highest value in either hand was used for the analyses. ${ }^{11}$ Muscle strength was considered low when HGS was $<28 \mathrm{~kg}$ in men, and $<18 \mathrm{~kg}$ in women.

\section{Gait Speed}

Physical performance was measured using a 4-m GS test. The participants were instructed to walk a $4 \mathrm{~m}$ straight course marked on the floor. There were no obstacles, and the participants were instructed to walk at their usual pace. They could use a walking aid, such as a cane, if necessary. Participants were given two opportunities to perform this test. The raw score was recorded as the number of seconds required to walk $4 \mathrm{~m}$ in each of the two tests, and the better GS was used for scoring. The cut-off value for GS for diagnosing sarcopenia was $<0.1 \mathrm{~m} / \mathrm{s}$ in men and women.

\section{Skeletal Muscle Mass Index}

To determine the SMI, bioelectrical resistance was obtained using an InBody 720 (Biospace Co., Ltd, Seoul, Korea) at frequencies of 5, 50, 250, and $500 \mathrm{kHz}$. The principle of bio-electrical impedance analysis (BIA) is a method of measuring body composition via the detected electrical resistance, by passing a very weak current through the body and measuring skeletal muscle mass and body fat mass. This device uses eight tactile electrodes, four in contact with the palm and thumb of both hands and four in contact with the anterior and posterior aspects of the soles of both feet. The participant stands on the soles of their feet, in contact with the foot electrodes, and grips the hand electrodes with both hands. The SMI was calculated by dividing the skeletal muscle mass by the square of the height $\left(\mathrm{kg} / \mathrm{m}^{2}\right)$; and low muscle mass was defined as an SMI $<7.0 \mathrm{~kg} / \mathrm{m}^{2}$ in men and $<5.7 \mathrm{~kg} / \mathrm{m}^{2}$ in women.

\section{Diagnosis of AWGS Sarcopenia}

Sarcopenia was defined according to the AWGS. ${ }^{3}$ The AWGS definition of sarcopenia combines low muscle mass and low muscle strength, and/or physical performance: HGS $<28 \mathrm{~kg}$ in men, $<18 \mathrm{~kg}$ in women and/or GS $<1.0 \mathrm{~m} / \mathrm{s}, \mathrm{SMI}<7.0 \mathrm{~kg} / \mathrm{m}^{2}$ in men, and $<5.7 \mathrm{~kg} / \mathrm{m}^{2}$ in women.

\section{Diagnosis of Simplified Algorithm A and B (with No Gait Speed Measure)}

Algorithm A first considers muscle strength and then, when this is low, muscle mass, ${ }^{12}$ while algorithm B first considers muscle mass and then, if this low, muscle strength. ${ }^{9}$ When both are applied, subjects with low muscle mass and strength are considered sarcopenic. All three algorithms employ the same methods to measure muscle mass and strength, only differing in the presence or absence of a gait speed component.

\section{Statistical Analysis}

Demographic characteristics of the community-dwelling older adults were analyzed by frequency, percentage, mean, and standard deviation (SD), according to sex. The means with SD were calculated for continuous variables and the frequencies were calculated as percentages for categorical variables. 
The Kolmogorov-Smirnov's test was used to determine were normally distributed. The verification of algorithms $\mathrm{A}$ and $\mathrm{B}$ was performed as follows. First, the difference between the three algorithms were identified using the chi-square test, and the prevalence of sarcopenia according to each algorithm was calculated. In addition, the specificity and sensitivity of algorithms A and B were confirmed. Statistical analyses were performed using IBM SPSS software (IBM SPSS Inc., Chicago, IL), and $p<0.05$ was considered statistically significant.

\section{Results}

This study included 388 community-dwelling older adults (254 women and 134 men) with a mean \pm SD age of 77.8 \pm 6.26 years (range, 65-93 years). Characteristics of the participants are reported in Table 1. Low muscle strength was present in $58.8 \%$, low GS in $93.0 \%$, and low gait speed in $97.2 \%$.

We also calculated the prevalence of sarcopenia using the muscle mass and strength algorithm, and compared the prevalence of sarcopenia determined using the algorithm including GS. No significant difference was found ( $p=0.157)$ between the prevalence of sarcopenia estimated with the AWGS-recommended algorithm (41.0\%) (Figure 1) and that estimated by algorithms A $(37.6 \%)$ or B $(37.6 \%)$ (Figures 2 and 3). Of the 388 subjects, 111 participants were diagnosed with sarcopenia using all three methods. In addition, the sensitivity of algorithms $\mathrm{A}$ and $\mathrm{B}$ was $91.0 \%$, and the specificity was $100 \%$.

\section{Discussion}

This study was conducted to diagnose sarcopenia of the community-dwelling older adults in the rural areas of Korea and to verify the effectiveness of using two simplified sarcopenia diagnosis algorithms based on the AWGSrecommended algorithm.

\section{Prevalence of Sarcopenia According to the AWGS Algorithm}

In this study, the prevalence of sarcopenia, as defined by the AWGS-recommended algorithm, in Korean rural community-dwelling older adults (mean age 77.80 \pm 6.26 years) was $41.0 \%$ overall $(40.3 \%$ in men, $41.3 \%$ in women). The prevalence of sarcopenia among communitydwelling older adults (770 men and 1000 women, aged $>65$ years) surveyed using the 2010-2011 Korean National Health and Nutrition Examination Survey was 6.6\% in men and $9.4 \%$ in women. ${ }^{13}$ In that study, sarcopenia was diagnosed based on a value derived by dividing the appendicular skeletal muscle mass (ASM), determined by dualenergy X-ray absorptiometry (DEXA), by weight and multiplied by 100 .

Table I Characteristics of Community-Dwelling Korean Older Adult Participants

\begin{tabular}{|c|c|c|c|}
\hline & Total $(n=388)$ & Female $(n=254)$ & Male $(n=134)$ \\
\hline Age & $77.80 \pm 6.26$ & $78.17 \pm 6.00$ & $77.09 \pm 6.69$ \\
\hline Weight & $56.33 \pm 10.50$ & $52.89 \pm 8.59$ & $62.86 \pm|0.7|$ \\
\hline Height & $155.27 \pm 9.97$ & $150.43 \pm 8.08$ & $164.43 \pm 6.05$ \\
\hline Skeletal Muscle Mass Index(SMI)(kg/m²) & $6.23 \pm 0.95$ & $5.79 \pm 0.73$ & $7.06 \pm 0.75$ \\
\hline$<7.0$ or $5.7 \mathrm{~kg} / \mathrm{m}^{2}$ (men or women) & $160(41.2)$ & $247(97.2)$ & $54(40.3)$ \\
\hline$\geq 7.0$ or $5.7 \mathrm{~kg} / \mathrm{m}^{2}$ (men or women) & $228(58.8)$ & $7(2.8)$ & $80(59.7)$ \\
\hline Grip strength(kg) & $10.66 \pm 4.40$ & $11.34 \pm 4.52$ & $9.38 \pm 3.85$ \\
\hline$<28$ or $18 \mathrm{~kg}$ (men or women) & $36 I(93.0)$ & $227(89.4)$ & $134(100)$ \\
\hline$\geq 28$ or $18 \mathrm{~kg}$ (men or women) & $27(7.0)$ & $27(10.6)$ & $0(0)$ \\
\hline Gait speed(m/s) & $0.4 I \pm 0.3 I$ & $0.44 \pm 0.35$ & $0.36 \pm 0.23$ \\
\hline$\leq 0.8 \mathrm{~m} / \mathrm{s}$ & $377(97.2)$ & $106(41.7)$ & $130(97.0)$ \\
\hline$>0.8 \mathrm{~m} / \mathrm{s}$ & $\mathrm{II}(2.8)$ & $148(58.3)$ & $4(3.0)$ \\
\hline \multicolumn{4}{|l|}{ MMSE } \\
\hline Severe cognitive impairment & $123(31.7)$ & $7 I(28.0)$ & $52(38.8)$ \\
\hline Mild cognitive impairment & $199(51.3)$ & 142(55.9) & $57(42.5)$ \\
\hline No cognitive impairment & $66(17.0)$ & $4 I(16.1)$ & $25(18.7)$ \\
\hline
\end{tabular}

Note: Values are mean \pm SD or $n(\%)$.

Abbreviation: MMSE, Mini-Mental State Examination. 


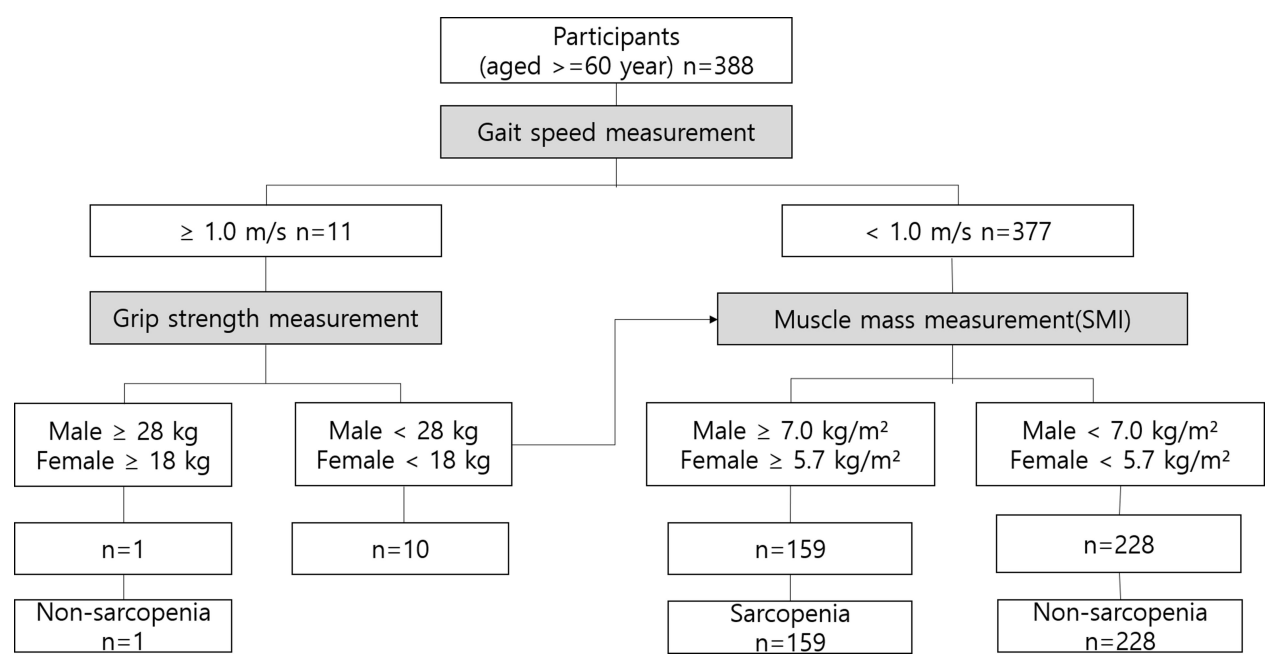

Figure I Assessment of prevalence of sarcopenia in community-dwelling older Korean according to the AWGS-recommended algorithm.

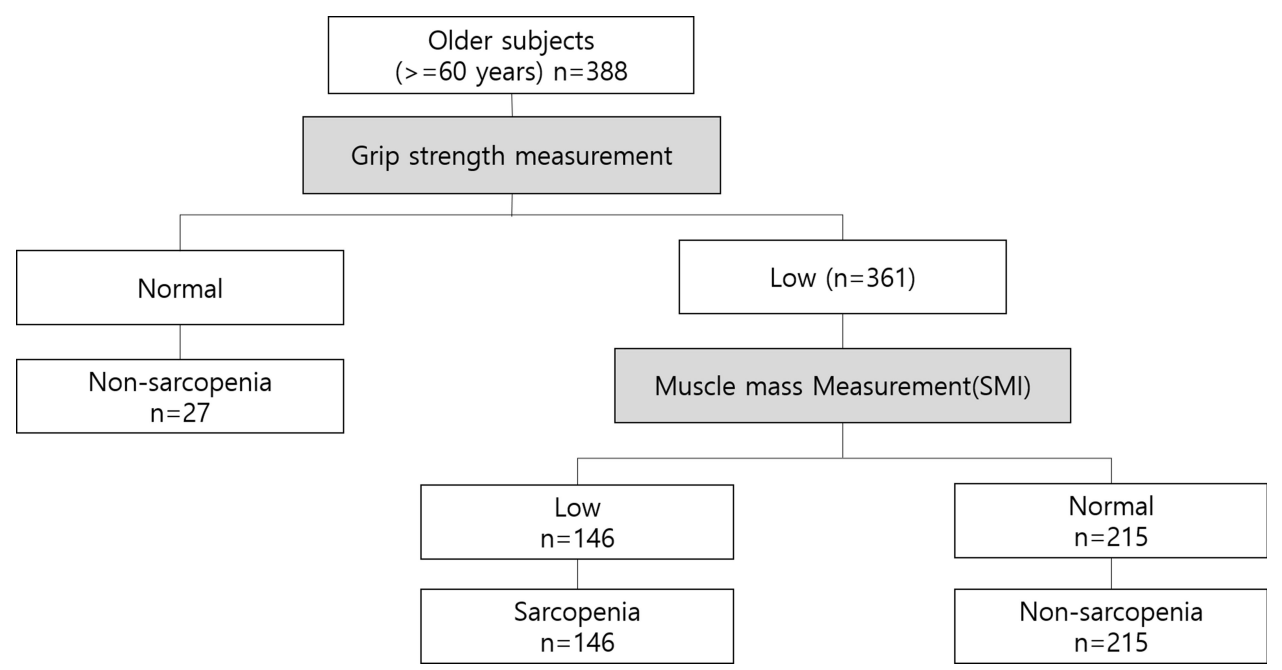

Figure 2 Assessment of prevalence of sarcopenia in community-dwelling older Korean according to the algorithm A, based on muscle strength and muscle mass.

In general, muscle mass is measured by DEXA, computed tomography, magnetic resonance imaging, and BIA. BIA is evaluated by dividing the ASM by the square of the height ${ }^{7}$ or by weight and multiplying by $100 .{ }^{14}$ BIA is widely used because it is a safe and cost-effective method that avoids radiation exposure and measures body composition. ${ }^{15}$ In our study, muscle mass was calculated by dividing the ASM by height squared, and muscle mass, muscle strength, and muscle function were all considered when diagnosing sarcopenia. Thus, an accurate comparison of the two studies is difficult because the muscle mass measurement method and the variables for diagnosing sarcopenia were applied differently.

Since muscle mass does not correlate positively with muscle function, muscle strength and physical function were also measured, unlike in previous studies in which sarcopenia was diagnosed only measuring muscle mass. ${ }^{16}$ For accurate comparisons, we searched for studies from other countries in Asia that applied the same measurement variables and cut-off values (HGS $<28 \mathrm{~kg}$ in men, $<18 \mathrm{~kg}$ in women, $\mathrm{GS}<1.0 \mathrm{~m} / \mathrm{s}$, and $/$ or $\mathrm{SMI}<7.0 \mathrm{~kg} / \mathrm{m}^{2}$ in men, $<5.7 \mathrm{~kg} / \mathrm{m}^{2}$ in women) used in this study. The prevalence of sarcopenia in 1076 community-dwelling older adults in China aged over 60 years (mean age $67.29 \pm 6.0$ years) was $9.3 \%$ in men, $6.4 \%$ in women, and $11.5 \%$ overall. ${ }^{17} \mathrm{In}$ another study from China, the prevalence of sarcopenia in 4500 community-dwelling older adults over the age of 50 years (mean age $62.4 \pm 8.3$ years) in four regions was $19.31 \%{ }^{6}$ In Japan, the prevalence of sarcopenia among 959 community-dwelling older adults (aged 60 years and older) was $9.6 \%$ in men and $7.7 \%$ in women. ${ }^{18}$ Previous 


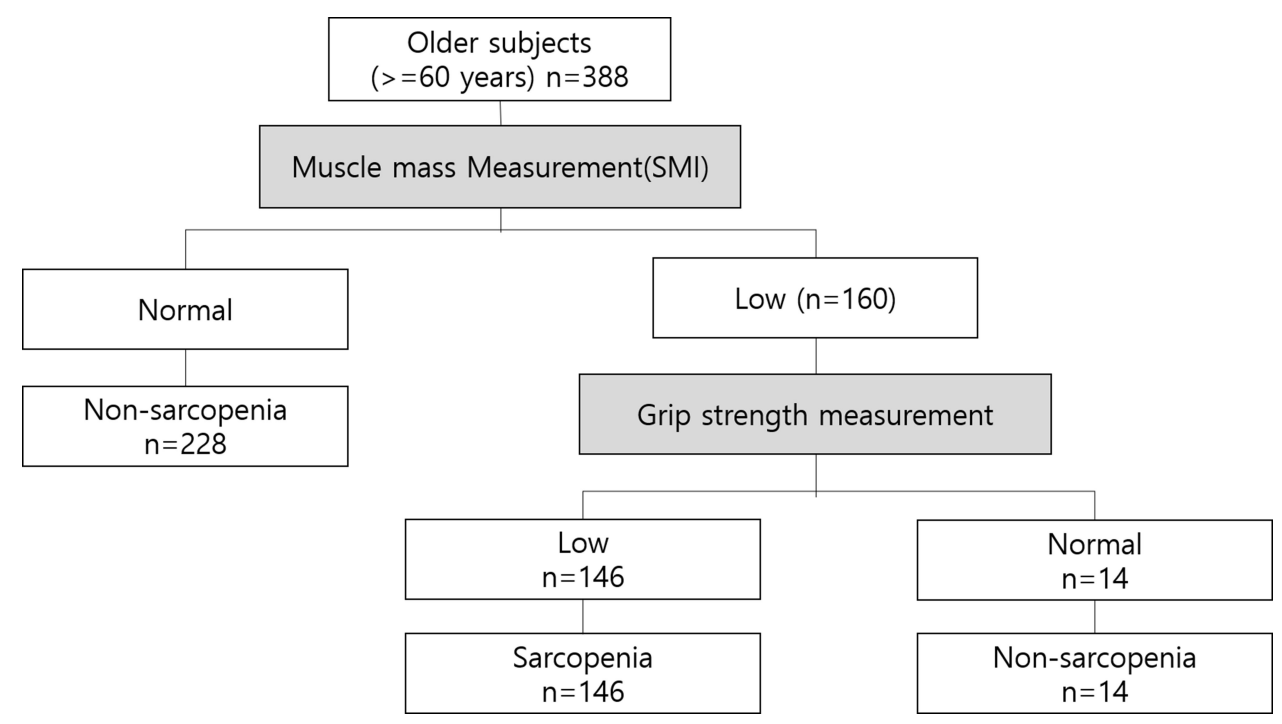

Figure 3 Assessment of prevalence of sarcopenia in community-dwelling older Korean according to the algorithm B, based on muscle mass and muscle strength.

studies on the prevalence of sarcopenia in communitydwelling older adults using the AWGS-recommended algorithm have reported values ranging from $9.3 \%$ to $19.31 \%{ }^{6,17,18}$

Although the AWGS algorithm had a high sensitivity and validity, the reason for the higher prevalence in this study than that in previous studies was probably the difference in demographic characteristics. The mean age of the subjects in Wang et $\mathrm{al}^{17}$ study was $67.29 \pm 6.0$ years, while it was $62.4 \pm 8.3$ years in Liu et $\mathrm{al}^{6}$ study. The mean age of the subjects in this study was $77.80 \pm 6.26$ years, which was slightly older than that in the other two studies. The prevalence of sarcopenia increases significantly with age in both men and women. ${ }^{19}$ In fact, according to Kim et al, ${ }^{13}$ adults aged $70-74$ years were at a 4.10 -fold higher risk of sarcopenia than those aged 65-69 years, and those aged 75-79 years were at a 3.15-fold higher risk of sarcopenia than those 65-69 years old, supporting the results of this study.

Hamyang-gun, the area in which data were collected in this study is a rural area where individuals aged 60 years and over account for $42.5 \%$ of the population. ${ }^{20}$ In our study, more than $44.6 \%$ of the subjects were aged over 80 years. It is estimated that the prevalence rate is higher than that of other rural areas, especially because it is reported that sarcopenia increases by $53.2 \%$ in individuals aged 80 years or over. There are currently countless studies that have examined the prevalence of sarcopenia. However, few of them included rural populations. We found only 3 studies that included elderly people who lived in rural communities. One study was conducted in Thailand; ${ }^{22}$ however, the prevalence of sarcopenia in rural elders was not reported. The other study was conducted in Taiwan, and the authors reported that the prevalences of sarcopenia and severe sarcopenia were $7.1 \%$ and $5.6 \%$ in rural elders, respectively. ${ }^{19}$ In China, a previous study using the AWGS algorithm found the prevalences of sarcopenia among older adults in rural and urban areas of $13.1 \%$ and $7.0 \%$, respectively. A multiple logistic regression revealed that rural residence was an independent risk factor for sarcopenia. ${ }^{23}$ This is because the development of science and technology related to agricultural production and the labour required for agriculture has significantly decreased, although chronic diseases are occurring as a result of agricultural-specific environments, such as working for a long time in an uncomfortable posture. ${ }^{24,25}$ In addition, the increased sedentary life due to the mechanization of agricultural equipment has increased the prevalence of chronic diseases, such as muscle mass reduction, excessive accumulation of body fat, cardiovascular disease, and metabolic syndrome in the elderly. ${ }^{26}$ Among them, the advent of musculoskeletal diseases cannot exclude the possibility of accelerating muscle degeneration since it reduces physical activity and restricts mobility. This means that old adults in rural areas may have different clinical characteristics compared to their counterparts living in cities; ${ }^{21}$ in the future, further studies are warranted in order to determine the prevalence of sarcopenia by focusing on older adults in rural areas. 
The main determinant of sarcopenia was muscle mass, given that $95.6 \%$ of participants were classified as having a low gait speed and $93 \%$ as having low muscle strength. It may possibly be argued that the cutoff points used in our study were too low. Indeed, low muscle strength of HGS in the AWGS is defined as $<28 \mathrm{~kg}$ in men and $<18 \mathrm{~kg}$ in women. However, there is no formal data regarding HGS for Koreans. Masanés et $\mathrm{al}^{27}$ demonstrated that changes in cutoff points for gait speed and grip strength have a limited effect on sarcopenia prevalence, while small changes in cut off points taken as reference points for muscle mass may have a substantial impact. These findings are consistent with the results of the BELFRAIL population-based study of the extremely old people ( $\geq 80 \mathrm{yrs})$, which reported that the prevalence of sarcopenia estimated was similar to that obtained using muscle mass as a single criterion. ${ }^{28}$ Yosida et al also found that only $3.7 \%$ of their study population had a gait speed of $\geq 0.8 \mathrm{~m} / \mathrm{s} .{ }^{12}$ They observed that gait speed and muscle strength may be limited by functional impairment and concluded that muscle mass measurements may be more valuable than functional measurements for sarcopenia diagnosis in populations whose function is severely compromised. Further research should examine the need to include gait speed in algorithms, in addition to the validity of cut-off values.

\section{Validation of Simplified Algorithms for Sarcopenia Diagnosis}

The most widely used tools for diagnosing sarcopenia in communities is the AWGS-recommended algorithm. BIA, hand grip dynamics, and GS are considered the most effective, reliable, and viable technologies for measuring algorithmic variables in Asia. ${ }^{3}$

No significant difference was found $(p=0.223)$ between the sarcopenia prevalence determined using the AWGSrecommended algorithm (41.0\%) and that estimated using algorithms A (37.6\%) or B (37.6\%), validating the use of these two simplified algorithms for sarcopenia diagnosis in this type of a population. With algorithm A, muscle strength is measured first, and muscle mass only needs to be assessed in women with muscle strength below $18 \mathrm{~kg}$ and in men with strength below $26 \mathrm{~kg}$. However, $93 \%$ of the present participants had muscle strength below these cut-off values; therefore, it was necessary to measure the muscle mass in almost all cases. Conversely, muscle mass is measured first in algorithm B, and muscle strength only needs to be assessed in individuals with low muscle mass (SMI
$<5.7 \mathrm{~kg} / \mathrm{m}^{2}$ in women and $<7.0 \mathrm{~kg} / \mathrm{m}^{2}$ in men). This proved to be an advantage in the present population, because the muscle mass measurement alone sufficed to rule out sarcopenia in 288 (59\%) of the participants.

Many previous studies have excluded individuals who could not walk or had a cognitive impairment, thereby limiting the representativeness of the results. ${ }^{22-26}$ The use of GS for the diagnosis of sarcopenia is problematic, and can lead to some degree of error in diagnosis. ${ }^{8}$ Omitting GS in algorithms A and B makes it quick and easy to diagnose sarcopenia in older community-dwelling people with marked limitations, particularly those who are not walking well, as suggested by Yoshida et al assumed. ${ }^{12}$

\section{Conclusion}

By using a questionnaire and physical measurements, this study sought to identify the prevalence of sarcopenia in community-dwelling Korean older adults, and concurrently to validate the use of the simplified algorithms A and B for diagnosing sarcopenia. The overall prevalence of sarcopenia, as determined by the AWGS-recommended algorithm and simplified algorithms $\mathrm{A}$ and $\mathrm{B}$, was highly similar (41.0\%, 37.6\% and $37.6 \%$, respectively). Thus, we have confirmed that the simplified algorithms are suitable for diagnosing sarcopenia in community-dwelling elderly individuals residing in rural Korea. The data obtained may serve as a basis for developing a simpler and more reliable diagnosis of sarcopenia in older adults with functional and/or cognitive impairment, as well as in nursing home residents.

The study was conducted with a limited number of participants, all of whom were rural community-dwelling older adults in Korea; thus, our findings may have limited generalizability. However, this study verified that the diagnosis of muscle sarcopenia can be easily delivered by measuring muscle mass using a BIA device, without using X-ray imaging with a wide range of measurements being limited. BIA devices are very sensitive to changes in moisture conditions and can overestimate fat levels, but many studies have found that this is a reasonable, reliable, and feasible way to evaluate muscle mass when diagnosing myasthenia. ${ }^{29-31}$ In addition, this study focused on adults dwelling in a single rural area in Korea; in the future, it will be necessary to conduct additional research to confirm the validity of algorithms for elderly living in various regions.

Furthermore, whether including gait speed is necessary when screening for sarcopenia in community-dwelling older adults remains debatable. Also, there has not been 
a paper that mentions the cost-effective part of diagnosing sarcopenia with a simplified algorithm. However, if the sarcopenia is diagnosed with a simplified algorithm that excludes GS, it will be able to save it compared to the existing cost, and the measurement time will be shortened and it will contribute to efficient diagnosis.

Future research should examine the necessity of including gait speed and HGS in algorithms, alongside the validity of cut-off values.

\section{Abbreviations}

ASM, appendicular skeletal muscle mass; AWGS, Asian Working Group for Sarcopenia; BIA, bio-electrical impedance analysis; BMI, body mass index; CT, computed tomography; DEXA, dual-energy X-ray absorptiometry; EWGSOP, European Working Group on Sarcopenia in Older People; GS, GS; HGS, hand grip strength; IWGS, International Working Group on Sarcopenia; KNHANES, Korean National Health and Nutrition Examination Survey; MMSE-K, Korean version of Mini-Mental State Examination; MRI, magnetic resonance imaging; SD, standard deviation; SMI, skeletal muscle mass index.

\section{Data Sharing Statement}

The data that support the findings of this study are available from the corresponding author on request.

\section{Ethics Approval and Informed Consent}

We received ethical approval for the study from the Institute of Review Board at Gyeongsang National University (Approval No. GIRB-A19-Y-0080); all participants were informed of the objectives, methods, and procedures of data collection. Their rights to confidentiality, anonymity, and voluntary withdrawal from study participation were explained and assured, as was the disposal of material containing personal information after the completion of the study. Written informed consent to participate was obtained from all participants.

\section{Acknowledgments}

We thank all the participants for the time dedicated to this study.

\section{Author Contributions}

All authors contributed to the study design, acquisition of data, and article preparation; took part in drafting the article or revising it critically for important intellectual content; agreed to submit to the current journal; gave final approval of the version to be published and agree to be accountable for all aspects of the work.

\section{Funding}

This work was supported by funding from the Research Promotion Program, Gyeongsang National University, 2019. The funding body had no role in the design of this study, in collection, analysis and interpretation of data, and in writing the manuscript.

\section{Disclosure}

The authors report no conflicts of interest in this work.

\section{References}

1. Statistical Office. Aging statistics for 2019 [Internet]; 2019. Available from: http://kostat.go.kr/portal/korea/kor_nw/1/6/1/index.board? bmode $=$ read $\&$ bSeq $=\& a S e q=377701 \&$ page $\mathrm{No}=1 \&$ row $\mathrm{Num}=$ $10 \&$ navCount $=10 \&$ currPg $=\&$ searchInfo $=\&$ sTarget $=$ title \&sTxt $=$. Accessed January 6, 2020.

2. Goates S, Du K, Arensberg MB, Gaillard T, Guralnak J, Pereira SL Economic impact of hospitalizations in us adults with sarcopenia. $J$ Frailty Aging. 2019;8(2):93-99. doi:10.14283/ jfa.2019.10.

3. Chen LK, Liu LK, Woo J, et al. Sarcopenia in Asia: consensus report of the asian working group for sarcopenia: 2019 consensus update on sarcopenia diagnosis and treatment. J Am Med Dir Assoc. 2020;21 (3):300-307. doi:10.1016/j.jamda.2019.12.012

4. Rodriguez-Rejon AI, Rodriguez-Rejon R, Artacgo A, Puerta A, Zuniga A, Ruiz-Lopez MD Diagnosis of sarcopenia in long-term care homes for the elderly: the sensitivity and specificity of two simplified algorithms with respect to the EWGSOP consensus. J Nutr Health Aging. 2018;22(7):796-801. doi:10.1007/s12603-0181004-x.

5. Mayhew AJ, Amog K, Phillips S, et al. The prevalence of sarcopenia in community-dwelling older adults, an exploration of differences between studies and within definitions: a systematic review and meta-analyses. Age Ageing. 2019;48(1):48-56. doi:10.1093/ageing/afy106.

6. Liu X, Hou L, Xia X, et al. Prevalence of sarcopenia in multi ethnics adults and the association with cognitive impairment: findings from West-China health and aging trend study. BMC Geriatr. 2020;20 (63):1-10. doi:10.1186/s12877-020-1468-5.

7. Baumgartner RN, Koehler KM, Gallagher D, et al. Epidemiology of sarcopenia among the elderly in New Mexico. Am J Epidemiol.1998;147(8):755-763. doi:10.1093/oxfordjournals.aje. a009520.

8. Bianchi L, Ferrucci L, Cherubini A, et al. The predictive value of the EWGSOP definition of sarcopenia: results from the in CHIANTI study. J Gerontol a Biol Sci Med Sci. 2016;71(2):259-264. doi:10.1093/gerona/glv129.

9. Rodríguez-Rejón AI, Ruiz-López MD, Artacho MLR Diagnosis and prevalence of sarcopenia in long-term care homes: EWGSOP2 versus EWGSOP1. Nutricion hospitalaria. 2019; 36(5), 1074-1080. doi:10.20960/nh.02573.

10. Kwon YC, Park JH Korean version of mini-mental state examination (MMSE-K) Part I : development of the test for the elderly $J$ Clin Neurol. 1989;1:123-135. 
11. Hofmann M, Schober-Halper B, Oesen S, et al. Effects of elastic band resistance training and nutritional supplementation on muscle quality and circulating muscle growth and degradation factors of institutionalized elderly women: the vienna active ageing study (VAAS). Eur J Appl Physiol. 2016;116(5):885-897. doi:10.1007/ s00421-016-3344-8.

12. Yoshida D, Suzuki T, Shimada H, et al. Using two different algorithms to determine the prevalence of sarcopenia. Geriatr Gerontol Int. 2014;14(S1):46-51. doi:10.1111/ggi.12210.

13. Kim HH, Kim JS, Yu JO Factors contributing to sarcopenia among community-dwelling older Korean adults. J Korean Gerontol Nurs. 2014;16(2):170-179. doi:10.17079/jkgn.2014.16.2.170.

14. Janssen I, Ross R Linking age-related changes in skeletal muscle mass and composition with metabolism and disease. J Nutr Health Aging. 2005;9(6):408-419.

15. Kuriyan R Body composition techniques. Indian $J$ Med Res. 2018;148(5):648-658. doi:10.4103/ijmr.IJMR_1777_18.

16. Cederholm TE, Bauer JM, Boirie Y, Schneider SM, Sieber CC, Rolland Y Toward a definition of sarcopenia. Clin Geriat Med. 2011;27(3):341-353. doi:10.1016/j.cger.2011.04.001.

17. Wang H, Hai S, Cao L, Zhou J, Liu P, Dong BR Estimation of prevalence of sarcopenia by using a new bioelectrical impedance analysis in Chinese community-dwelling elderly people $B M C$ Geriatr. 2016;16(1):216. doi:10.1186/s12877-016-0386-z.

18. Yuki A, Ando F, Otsuka R, Matsui Y, Harada A, Shimokata H Epidemiology of sarcopenia in elderly Japanese. J Phys Fit Sports Med. 2015;4(1):111-115. doi:10.7600/jpfsm.4.111.

19. Wu CH, Chen KT, Hou MT, et al. Prevalence and associated factors of sarcopenia and severe sarcopenia in older Taiwanese living in rural community: the Tianliao old people study 04. Geriatr Gerontol Int. 2014;14(S1):69-75. doi:10.1111/ggi.12233.

20. Hamyang-gun Office. Population status by age (foreigners excluded) [Internet]; 2020. Available from: http://www.hygn.go.kr/country/ 00780/04266.web. Accessed January 6, 2020.

21. Meng $\mathrm{P}, \mathrm{Hu} \mathrm{YX}$, Fan L, et al. Sarcopenia and sarcopenic obesity among men aged 80 years and older in Beijing: prevalence and its association with functional performance. Geriatr Gerontol Int. 2014;14(1): 29-35. doi:10.1111/ggi.12211
22. Pongchaiyakul C, Limpawattana P, Kotruchin P, Rajatanavin R Prevalence of sarcopenia and associated factors among Thai population. J Bone Miner Metabolis. 2013;31(3):346-350. doi:10.1007/s00774-013-0422-4.

23. Gao L, Jiang J, Yang M, Hao Q, Luo L, Dong B Prevalence of sarcopenia and associated factors in Chinese community-dwelling elderly: comparison between rural and urban areas. J Am Med Dir Assoc. 2015;16(11):1003.e1-e6. doi:10.1016/j.jamda.2015.07.020.

24. Park TJ, Kim BS, Chon HJ Factors associated with farmers' Syndrome. Korean J Rural Med.1994;19(1):5-13.

25. Holmberg S, Thelin A, Stiernstrom E, Svardsudd K The impact of physical work exposure on musculoskeletal symptoms among farmers and rural non-farmers. Ann Agric Environ Med. 2003;10 (2):179-184.

26. Jenkins PL, Earle-Richardson G, Bell EM, May JJ, Green A Chronic disease risk in central. New York dairy farmers: results from a large health survey. Am J Ind Med. 2004;47(1):20-26. doi:10.1002/ ajim. 20110.

27. Masanés F, Rojano I, Luque X, et al. Cut-off points for muscle mass not grip strength or gait speed - determine variations in sarcopenia prevalence. J Nutr Health Aging. 2017;21(7):825-829. doi:10.1007/ s12603-016-0844-5.

28. Legrand D, Vaes B, Matheï C, Swine C, Degryse JM The prevalence of sarcopenia in very old individuals according to the European consensus definition: insights from the BELFRAIL study. Age Ageing. 2013;42(6):727-734. doi:10.1093/ageing/aft128.

29. Dodds RM, Granic A, Davies K, Kirkwood TB, Jagger C, Sayer AA Prevalence and incidence of sarcopenia in the very old: findings from the Newcastle 85+ study. J Cachexia Sarcopenia Muscle. 2016;8 (2):229-237. doi:10.1002/jcsm.12157.

30. Yilmaz O, Bahat G Suggestions for assessment of muscle mass in primary care setting. Aging Male. 2017;20(3):168-169. doi:10.1080/ 13685538.2017 .1311856$.

31. Ibrahim K, Howson FFA, Culliford DJ, Sayer AA, Roberts HC The feasibility of assessing frailty and sarcopenia in hospitalised older people: a comparison of commonly used tools. BMC Geriatr. 2019;19(1):42. doi:10.1186/s12877-019-1053-y.
Clinical Interventions in Aging

\section{Publish your work in this journal}

Clinical Interventions in Aging is an international, peer-reviewed journal focusing on evidence-based reports on the value or lack thereof of treatments intended to prevent or delay the onset of maladaptive correlates of aging in human beings. This journal is indexed on PubMed Central, MedLine, CAS, Scopus and the Elsevier
Bibliographic databases. The manuscript management system is completely online and includes a very quick and fair peer-review system, which is all easy to use. Visit http://www.dovepress.com/ testimonials.php to read real quotes from published authors. 